\title{
Publizieren in deutsch - attraktiv, sinnvoll, unabdingbar
}

\section{Publish in German - Attractive, Meaningful, Indispensable}

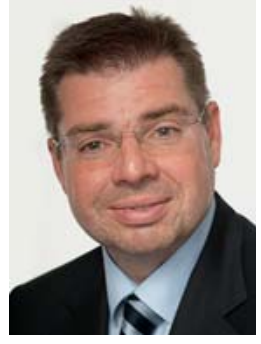

D. C. Wirtz

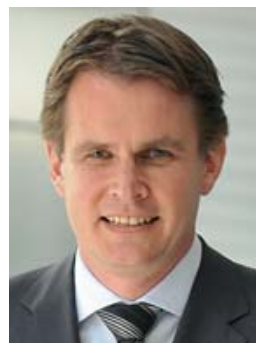

U. Stöckle

\section{Bibliografie}

DOI http://dx.doi.org/

$10.1055 / \mathrm{s}-0032-1328246$

Z Orthop Unfall 2013; 151: 13

(c) Georg Thieme Verlag KG

Stuttgart · New York .

ISSN 1864-6697

\section{Korrespondenzadressen Univ.-Prof. Dr. Ulrich Stöckle Ärztlicher Direktor \\ BGU Klinik Tübingen \\ Schnarrenbergstraße 96 \\ 72076 Tübingen \\ Tel.: 0 07071/606-1001 \\ Fax: 07071/606-1002 \\ ustoeckle@bgu-tuebingen.de}

\section{Univ.-Prof. Dr. med}

Dieter C. Wirtz

Direktor der Klinik und

Poliklinik für Orthopädie

und Unfallchirurgie

Universitätsklinikum Bonn

Sigmund-Freud-Straße 25

53105 Bonn

Tel.: 0228/287-14170

Fax: 0228/287-14175

dieter.wirtz@ukb.uni-bonn.de

\section{Liebe Leserinnen und Leser,}

wie schon in unserem Editorial 4/2012 ausgeführt ist dank der qualitativ hochwertigen Manuskripte, die aus unserer Zeitschrift in den Jahren 2009 und 2010 zitiert wurden, der Impact-Faktor 2011 auf 0,522 (0,343 in 2010) gestiegen. Für 2012 liegt der Index noch nicht vor, jedoch ist davon auszugehen, dass er weiterhin „nach oben“geht. Dieser Trend ist natürlich sehr erfreulich und hat dazu geführt, dass die Zeitschrift für Orthopädie und Unfallchirurgie im internationalen Ranking zwischenzeitlich unter den ersten 50 Journals in der Fachkategorie „Orthopedics" gelistet ist.

Wohlwissend, dass die internationale Wissenschaftssprache Englisch ist, und von einigen Wissenschaftlern die Meinung vertreten wird, dass derjenige, der nicht in Englisch publiziert, kein relevantes Wissen zu vermitteln habe, wollen wir auch in $\mathrm{Zu}$ kunft die deutsche Sprache in unserer Zeitschrift beibehalten und pflegen. Dies ist insbesondere unter der im Deutschen Ärzteblatt publizierten Erkenntnis begründet, dass $80 \%$ der deutschen Ärzte keine englischen Artikel lesen [1]. Nach einer Studie von Haße und Fischer in der Deutschen Medizinischen Wochenschrift [2] erwartet die überwiegende Mehrzahl an Ärzten deutschsprachige Artikel in den Zeitschriften ihrer Fachgesellschaft. Wir brauchen daher für die breite medizinische Versorgung, und insbesondere zur Erhaltung bzw. Verbesserung der notwendigen Versorgungsqualität in Orthopädie und Unfallchirurgie, dringend auch weiterhin deutschsprachige attraktive Zeitschriften, in denen bewährte Methoden einerseits, aber auch neueste wissenschaftliche Erkenntnisse andererseits zur Fort- und Weiterbildung angeboten werden.

Dem Anspruch der „Wissensvermittlung in der Breite" wollen wir mehr wie bisher Rechnung tragen durch eingeladene Übersichtsarbeiten und durch die Zusammenstellung von Schwerpunktheften zu wichtigen Themen unseres Faches. Auch Veränderungen der politischen Rahmenbedingungen, die von nationaler Relevanz in den verschiedenen Versorgungssektoren sind, sollen künftig mehr Berücksichtigung finden. Aber auch entsprechend der Tradition unserer wissenschaftlichen Zeitschrift wollen und müssen wir für die „Wissensvermittlung in der Tiefe“ attraktiv sein. Forschungsprojekte und deren Ergebnisse aus dem deutschsprachigen Sprachraum sollten in der Muttersprache publiziert werden, weil nur diese wirklich die Detailgenauigkeit und „Tiefe“ von Forschung ausdrücken lässt. Gerade wo die Beschreibung und Benennung komplexer Zusammenhänge von höchster Wichtigkeit ist, macht das Publizieren in präziser und reflektierter Form (ohne Übersetzungsbüro) Sinn.

Das Problem der „Wissenschaft in eigener oder fremder Sprache“ adressiert der Linguist und Sprachdidaktiker P. Frath in Forschung \& Lehre 1/2013 [3] sehr eindrücklich. Nach seiner Beobachtung ist es in Frankreich während der letzten Jahrzehnte zu einem massiven Einbruch der Verwendung des Französischen in der Forschung gekommen. Durch die immer breitere Anwendung des Englischen verliere die eigene französische Sprache immer mehr an Worten, in denen sie ihr Wissen ausdrücken könne. Dies gelte aber nicht nur für das Sprechen, sondern vor allem auch für das wissenschaftliche Schreiben. Wenn nicht mehr in der eigenen Sprache geschrieben werde, um höhere Bildung und innovative Forschungsergebnisse zu vermitteln, so werde sich dies auch auf den „Kulturwert Wissenschaft“ in der eigenen Gesellschaft auswirken.

Gewiss müssen französische wie auch deutsche Wissenschaftler weiterhin in Englisch publizieren. Nur so ist internationale Kommunikation und wissenschaftliche Diskussion möglich. Insbesondere für jüngere Kollegen mit dem Ziel der Habilitation an einer deutschen Universität spielt aber auch der in der Regel höhere Impact-Faktor in englischsprachigen Zeitschriften eine wesentliche Rolle. Hier werden unabhängig von der originären Qualität der Beiträge allein schon durch die höheren Auflagen mit einer größeren Leserschaft die publizierten Manuskripte häufiger zitiert, was die Impact-Faktor-abhängige Effizienz auf dem Weg der Habilitation deutlich erhöht. Allerdings ist auf dem Karriereweg auch zu bedenken, dass man national vornehmlich dann bekannt wird und entsprechende Themen „mitbesetzt“, wenn man in der oder den anerkannten Zeitschriften der Fachgesellschaften publiziert. Ein nicht ganz unerheblicher Faktor, wenn es um die Besetzung leitender Positionen in Kliniken geht. Wir sollten uns daher eine deutsche Schreib- und Publikationskultur erhalten. Die Zeitschrift für Orthopädie und Unfallchirurgie setzt als offizielles Organ unserer Fachgesellschaften auf eine sinnvolle Kombination von Beiträgen zur Berufspolitik, aus der Grundlagen- und klinischen Studienforschung, sowie auf gute Übersichtsarbeiten, die in ihrer Qualität den internationalen Vergleich nicht scheuen müssen. Mit diesem Strategiekonzept sehen wir als Herausgeber und Schriftleiter sehr positiv „nach vorne“. In deutscher Sprache zu publizieren und zu lesen, wird attraktiv, sinnvoll und unabdingbar bleiben.

\section{C. Wirtz}

\section{U. Stöckle}

\section{Literatur}

1 Krüger-Brand $H$. Patienteninformation: navigieren durchs Gesundheits-Web. Dtsch Aerztebl 2012; 109: A-1924/B-1593/C-1565

2 Haße W, Fischer RJ. Ärzteschaft gegen Anglisierung der Medizin. Dtsch Med Wochenschr 2003; 128: 1338-1341

3 Frath $P$. Unbequem, ungerecht und gefährlich. Zur Wissenschaftssprache Englisch in Frankreich und Europa. Forschung \& Lehre 2013; 1: 26-28

4 Kusch R, Beckmann A. Deutsch als Wissenschaftssprache (20.1.2013). Im Internet: www.dradio.de/dlf/ sendungen/studiozeit-ks/1034170; Stand: 20.1.2013 